\title{
Large Field Events in the Distant Magnetotail During Magnetic Storms
}

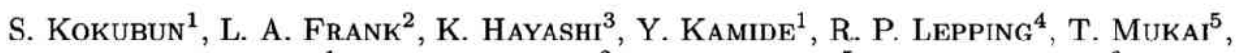 \\ R. NAKAmura ${ }^{1}$, W. R. Paterson ${ }^{2}$, T. Yamamoto ${ }^{5}$, and K. YUMOto ${ }^{6}$ \\ ${ }^{1}$ Solar-Terrestrial Environment Laboratory, Nagoya University, Toyokawa 442, Japan \\ ${ }^{2}$ Department of Physics and Astronomy, The University of Iowa, IA 52242, U.S.A. \\ ${ }^{3}$ Department of Earth and Planetary Physics, University of Tokyo, Tokyo 113, Japan \\ ${ }^{4}$ Laboratory for Extraterrestrial Physics, NASA/GSFC, Greenbelt, MD 20771, U.S.A. \\ ${ }^{5}$ Institute of Space and Astronautical Science, Sagamihara 229, Japan \\ ${ }^{6}$ Department of Physics, Kyushu University, Fukuoka 810, Japan
}

(Received December 12, 1995; Revised March 5, 1996; Accepted March 27, 1996)

\begin{abstract}
Although the magnetic field intensity in the lobe of the magnetotail is usually in the range of 7-10 $\mathrm{nT}$ at distances beyond $150 R_{E}$ as observed by ISEE 3 and GEOTAIL, it becomes at times more than $30 \mathrm{nT}$. The magnetic field of as large as $53 \mathrm{nT}$ was observed in the distant tail lobe by GEOTAIL at $-182 R_{E}$ on March 9,1993 , when a moderate magnetic storm was in progress. From the scan of daily plots of magnetic field data during the two year period of the GEOTAIL observations, intervals were chosen when the lobe field strength exceeding $20 \mathrm{nT}$ was registered. Large field events are found to mostly occur during a growing stage of the ring current. Events observed beyond $100 R_{E}$ downstream from the earth are associated with intermittent and temporal excursions of the spacecraft into the magnetotail. The characteristic time scale of magnetospheric period is approximately 20 minutes. These entries into the magnetotail are usually preceded by a strong southward component of sheath magnetic field. Since magnetic storms are associated with enhanced solar wind flux and IMF conditions, reconfiguration of the magnetotail due to increases of the total pressure in the sheath and directional changes of the sheath plasma flow should control the probability of spacecraft residence in the magnetotail. It is confirmed that the pressure balance approximately holds at the tail magnetopause under enhanced solar wind conditions by using high resolution plasma data. Observations of large lobe field events suggest that additional magnetic fluxes caused by the merging process near the earth during magnetic storms constrain the magnetotail radius from decreasing the nominal value significantly, although the magnetotail is in a compressed state due to the increase in exterior pressure.
\end{abstract}

\section{Introduction}

The ISEE 3 observation of the distant magnetotail has revealed that the lobe field magnitude is approximately $9 \mathrm{nT}$ at ISEE 3 apogee distances near $230 R_{E}$ (Slavin et al., 1985). It has also been reported that the tail has ceased expanding in radius at $230 R_{E}$ (Baker et al., 1987). Thus the tail boundary should be aligned parallel to the solar wind flow, at least on average. Fairfield (1993) has shown that the magnetotail configuration is controlled not only by solar wind flow and temperature, but also by the interplanetary magnetic field (IMF) and has suggested that very northward IMF can eliminate the extended tail. GEOTAIL observations support this suggestion that a very different configuration occurs when the IMF remains very northward for periods of at least several hours (Fairfield et al., 1996).

Concerning other aspects of the tail configuration, the effect of IMF $B_{y}$ have been studied extensively (e.g. Sibeck et al., 1985a, b; Sibeck et al., 1986; Owen et al., 1995). When the IMF $B_{y}$ is positive, the sense of torque will twist the north lobe toward the dawn flank and toward the dusk flank for the negative $B_{y}$ (Cowley, 1981). It has been reported that ISEE 3 observations are consistent with a sense predicted from tail twisting models (e.g. Sibeck et al., 1986). 
GEOTAIL was controlled by the lunar double swingby maneuvers until October, 1994 since its launch in July, 1992. On the basis of GEOTAIL magnetic field observations in the distant tail during the first one year period (Yamamoto et al., 1994a), the average profile of the magnetic field strength in the distant tail lobe is confirmed to fit approximately to the power law, $B(X)=$ $125 \times|X|^{-0.53}\left(X \leq-130 R_{E}\right)$, derived from the ISEE 3 observation by Slavin et al. (1985). Yamamoto et al. showed that the flaring angle of magnetic field lines in the tail lobe is statistically larger than the expansion angles of the tail magnetopause inferred from observed magnetopause location, suggesting that the lobe magnetic field lines are linked with those in the magnetosheath across the magnetopause. The GEOTAIL observations further suggest that the tail lobe is rich in cold plasma, which would directly enter the magnetotail (Mozer et al., 1994; Yamamoto et al., 1994b; Hirahara et al., 1995). Yamamoto et al. (1994a) have also reported that GEOTAIL occasionally observes large lobe magnetic fields of more than $20 \mathrm{nT}$. The largest magnitude of 53 $\mathrm{nT}$ in the tail lobe was recorded on March 9, 1993 when a moderately large magnetic storm was in progress (Kokubun et al., 1994b). In the analysis of the ISEE 3 observations Fairfield (1993) has presented simultaneous IMF 8 and ISEE 3 data for five geomagnetically disturbed intervals. During these periods, ISEE 3 remained within the magnetosheath for extended intervals, even when it was very near the center of an average tail. Fairfield suggested that nonradial solar wind flow associated with interacting solar wind streams moves a compressed tail away from the nominal position at these times. We notice in these data that large field magnitudes of more than $20 \mathrm{nT}$ were observed during temporal entries of the ISEE 3 spacecraft into the magnetotail. Fairfield (1993), however, was concerned the reconfiguration of the magnetotail during a sustained northward IMF. Dynamics of the magnetotail during geomagnetically disturbed intervals has not yet been examined in detail.

In this paper we will examine large lobe field events and their relations to solar wind and IMF conditions and ground magnetic activity. The observations indicate that large magnetic field events in the tail lobe are associated with dynamic behavior of the magnetotail during magnetic storms.

This study primarily uses the data obtained by the magnetic field experiment (MGF) onboard the GEOTAIL spacecraft for the survey of large field events in the distant tail lobe (Kokubun et al., 1994a). We mainly use spin averages of vector magnetic field data ( $\sim 3 \mathrm{sec}$.). The analysis also includes the plasma data, ion density and temperature, and components of bulk flow velocity, as measured with the Comprehensive Plasma Instrument (CPI) (Frank et al., 1994) and the Low Energy Particle (LEP) experiment (Mukai et al., 1994b). Since solar wind and IMF data from IMP 8 are available for a few intervals selected here, we refer to the Dst index as a measure of solar wind activity. To examine ground magnetic activity further we use magnetograms from the 210 meridian chain (Yumoto et al., 1992) and from the STEP Polar Network of University of Tokyo.

\section{Observations}

From the survey of daily plots of magnetic field data obtained in the distant tail epoch of two years we selected intervals when the total intensity of magnetic field in the magnetotail was larger than $20 \mathrm{nT}$. In Table 1 are listed positions of GEOTAIL in GSM coordinates and the corresponding Dst index for these large field events. It is noted that the field magnitude sometimes exceeds $30 \mathrm{n}$ T at a position of $X_{G S M}=-200 R_{E}$ in the distant tail. Out of twenty three intervals seventeen intervals are associated with $D s t$ indices of less than $-50 \mathrm{nT}$.

Orbits of GEOTAIL in these intervals are plotted on daily basis in Fig. 1. We used the modified GSM coordinates (hereafter referred to as $X^{\prime}, Y^{\prime}$, and $Z^{\prime}$ ) in this plot. In this coordinate system, we assume that the average solar wind aberration angle is $4^{\circ}$ (the corresponding solar wind speed is about $430 \mathrm{~km} / \mathrm{s}$ ) and that the hinging distance for the magnetospheric equatorial 
Table 1. Large lobe field events $(B>20 \mathrm{nT})$.

\begin{tabular}{|c|c|c|c|c|}
\hline Year & Date & $\begin{array}{r}\text { Dst index } \\
(\min ) n T\end{array}$ & $\begin{array}{l}\text { Position of GEOTAIL } \\
\text { in GSM }\left(R_{\mathbf{r}}\right)\end{array}$ & $\begin{array}{l}\text { Lobe magnetic } \\
\text { field (max) nT }\end{array}$ \\
\hline 1992 & November 9 & -93 & -68 & 26 \\
\hline 1993 & January 19 & -23 & -89 & 25 \\
\hline 1993 & January 25 & -47 & -94 & 34 \\
\hline 1993 & January 31 & -79 & -51 & 32 \\
\hline 1993 & February 7 & -60 & -85 & 27 \\
\hline 1993 & February 17 & -108 & -148 & 30 \\
\hline 1993 & February 20 & -53 & -161 & 22 \\
\hline 1993 & March 9 & -138 & -182 & 53 \\
\hline 1993 & March 11 & -120 & -181 & 21 \\
\hline 1993 & April 5 & -169 & -85 & 32 \\
\hline 1993 & May 27 & -44 & -188 & 24 \\
\hline 1993 & June 4 & -68 & -202 & 23 \\
\hline 1993 & June 23 & $>0$ & -208 & 28 \\
\hline 1993 & August 16 & -60 & -68 & 32 \\
\hline 1993 & September 13 & -169 & -68 & 28 \\
\hline 1993 & Oetober 9 & -85 & -145 & 27 \\
\hline 1993 & October 27 & -77 & -198 & 20 \\
\hline 1993 & November 3 & -116 & -207 & 30 \\
\hline 1993 & November 18 & -78 & -211 & 32 \\
\hline 1994 & January 11 & -37 & -90 & 32 \\
\hline 1994 & April 17 & -203 & -197 & 27 \\
\hline 1994 & July 14 & -12 & -119 & 26 \\
\hline 1994 & October 3 & -96 & -159 & 22 \\
\hline
\end{tabular}

plane is $10 R_{E}$. It is noticeable that large lobe field events were observed within locations of $Y^{\prime}= \pm 35 R_{E}$ in the region beyond $X^{\prime}=-50 R_{E}$. Several events are observed outside the nominal magnetotail.

Figure 2 shows nine examples of Dst index variations corresponding to intervals of large field events. In Fig. 2 intervals when the magnitude of lobe magnetic field exceeded $20 \mathrm{nT}$ are indicated by thick lines. It is evident that the majority of events occurs during the growing stage of the ring current. We, however, note that GEOTAIL did not always stay in high field tail lobe during the whole interval indicated by the thick line. Most events were observed in association with a temporal residence of the spacecraft in the magnetotail. We found that durations of large field events in the magnetotail range from several minutes to several hours. The largest field magnitude of the tail lobe, as observed by GEOTAIL in the distant tail survey for two years, was recorded at $X_{G S M}=-182 R_{E}$ on March 9, 1993, when a moderate magnetic storm was in progress. Figure 3 shows orbits of two intervals in the $Y^{\prime}-Z^{\prime}$ plane for which data are examined in detail below.

\subsection{March 8-9, 1993}

Figure 4 shows magnetic field and plasma data obtained by GEOTAIL on March 8-9, 1993. In Fig. 4 time for GEOTAIL data is shifted ahead by 33 minutes to account for an apparent convection time from IMP 8 to GEOTAIL. A storm sudden commencement occurred at 2137 UT on March 8 . The corresponding interplanetary shock was identified at 2134 UT in the IMP-8 magnetic field data. IMP-8 was located at $(7.0,16.1,30.1) R_{E}$ in GSM coordinates at that time. A sudden change of sheath magnetic field was observed with GEOTAIL at a distance of 


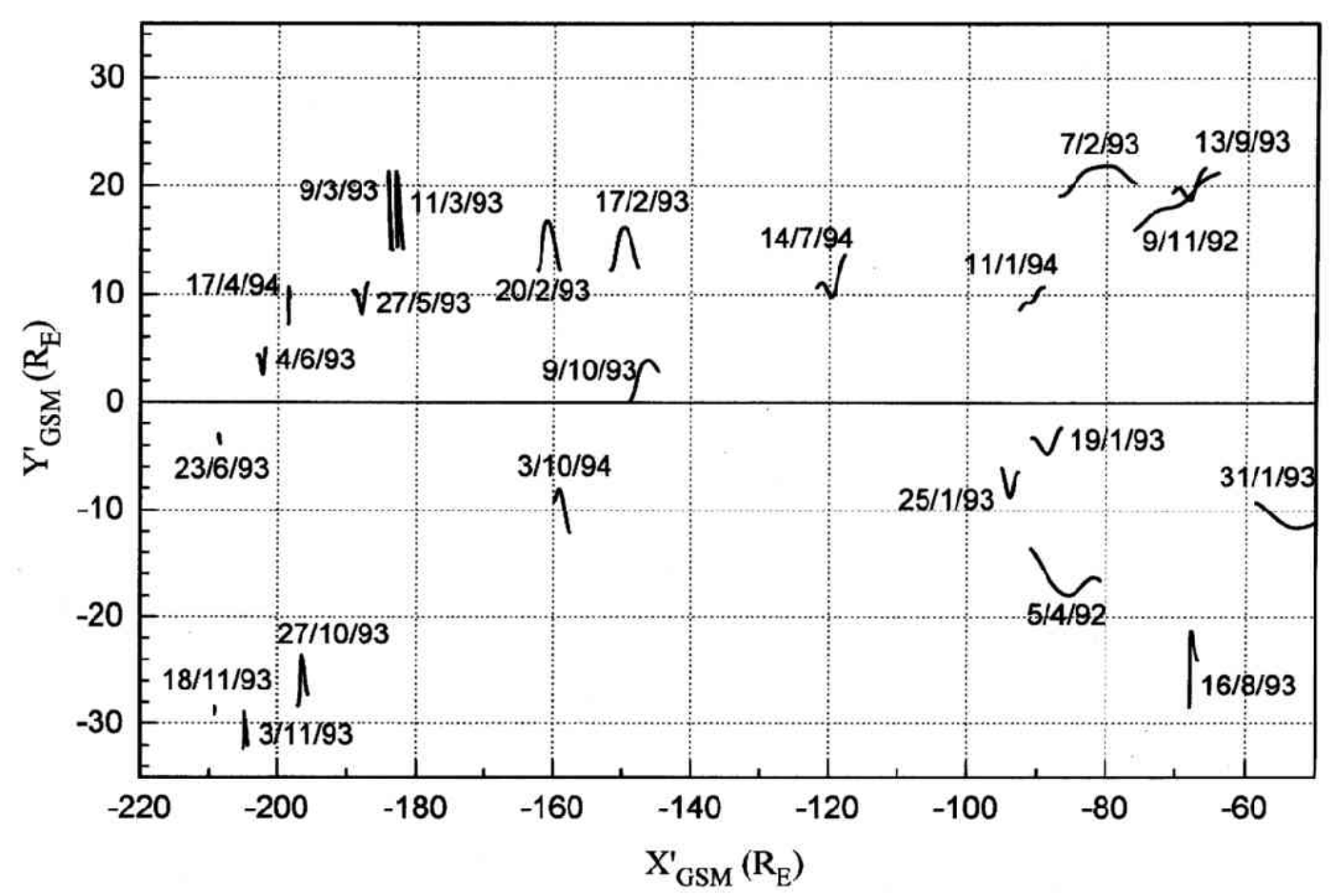

Fig. 1. Orbits of GEOTAIL for events with field magnitudes of more than $20 \mathrm{nT}$ in the tail lobe. Plots were made by daily basis in the modified GSM coordinates.

$X_{G S M}=-182.5 R_{E}$ at 2208:10 UT. In comparing the magnetic field data from GEOTAIL and IMP- 8 we note that the variation in sheath field observed by GEOTAIL were strikingly similar to IMF magnetic variation, although the field magnitude in the distant sheath was slightly smaller than that of IMF.

A series of GEOTAIL entries into the magnetotail were observed in intervals of 0112-29, 0147-0210, 0228-0238, 0406-15, 0440-53 and 0529-0714, as indicated by dashed lines in Fig. 4. These tail entries of the spacecraft are evidently identified by large deviations of GEOTAIL magnetic field data from IMF values. These entries, except the last one, occurred during an interval of negative $B_{y}$ of $\sim-20 \mathrm{nT}$.

During the interval of interest here GEOTAIL was located at positions of $Z^{\prime}=20-27 R_{E}$ outside the expected position of the magnetopause, as depicted in Fig. 3 (Yamamoto et al., 1994a). The largest value of the lobe magnetic filed, $53.0 \mathrm{nT}$, in the distant tail epoch of the GEOTAIL observations was recorded at 0121:03 UT during the first lobe entry on March 9. It is important to note that the $B_{z}$ components of both the IMF and sheath magnetic fields were southward during certain intervals before the lobe entries of GEOTAIL. The series of tail entries occurred during a growing stage of ring current activity associated with the southward component of IMF, as has already been mentioned.

Figure 5 displays CPI data, the flow speed, the flow angles to the GSM tail axis, the ion temperature, the density, the dynamic pressure $\left(N_{P} m V^{2}\right)$, and the total pressure $\left(2 N_{P} k T_{P}+\right.$ $B_{T}^{2} / 2 \mu$ ), together with magnetic field data for the five hour interval, when large lobe fields of more than $30 \mathrm{nT}$ were intermittently observed. Here, we assume in the total pressure estimation that ion and electron temperatures are equal. High density $\left(20 \sim 35 / \mathrm{cm}^{3}\right)$ and fast tailward flows 


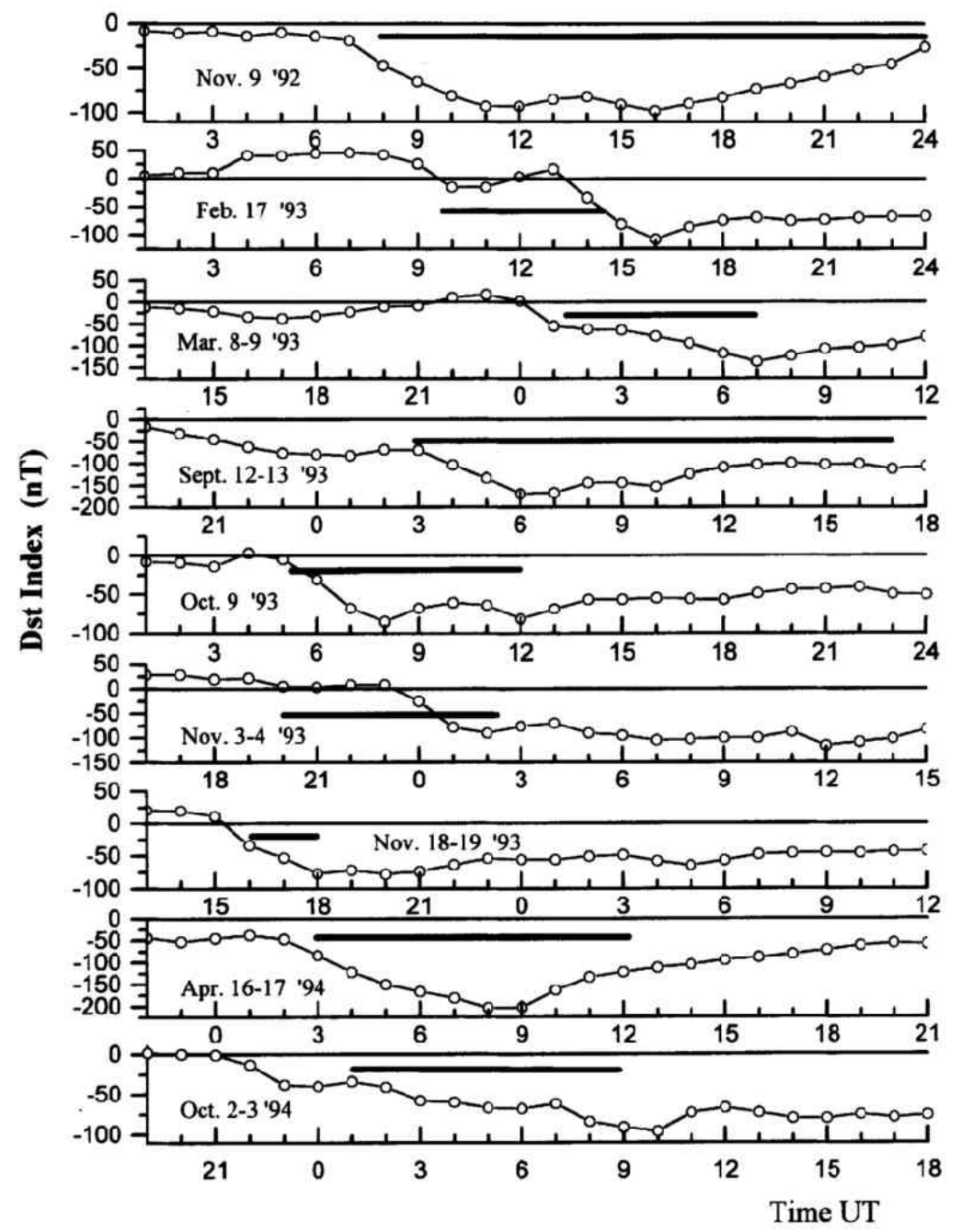

Fig. 2. Dst indices and intervals of large lobe fields indicated by thick lines as observed with GEOTAIL.

$\left(V_{x}=\sim 600 \mathrm{~km} / \mathrm{s}\right)$ were observed in the magnetosheath throughout this interval. The signatures of the tail lobe and the plasma sheet are clearly identified by the plasma data. Plasma sheet crossings from the north lobe to the south lobe were observed even though the spacecraft was located far from the $Y^{\prime}$ axis.

Figure 5 also reveals that the total pressure is almost continuous across the magnetopause and is unusually high, being approximately 30 times typical values of $0.02-0.03 \mathrm{nPa}$ in the solar wind (Fairfield, 1993). Thus, the magnetotail should be in a compressed state compared with the average condition. Assuming that a circular tail is confined by the magnetosheath pressure and that the average tail flux is conserved, we find that tail radius $(R)$ has a 1/4 power dependence on the external pressure $(P)$ (Fairfield, 1993):

$$
R=\left(P_{0} / P\right)^{1 / 4} R_{0}
$$

Observed external pressures are as large as $0.52-0.99 \mathrm{nPa}$ for eleven crossings of the magnetotail 


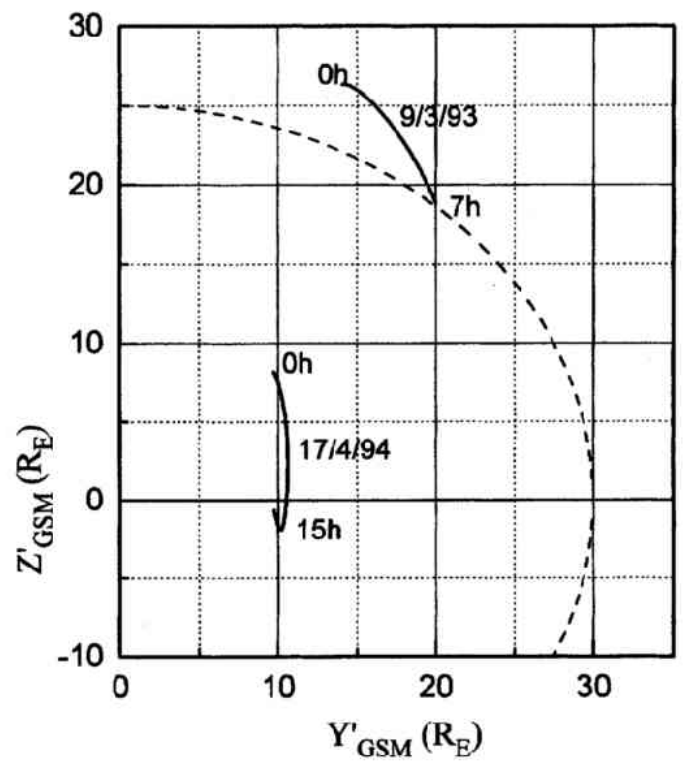

Fig. 3. Orbits of two intervals in the $Y^{\prime}-Z^{\prime}$ plane on March 9, 1993 and April 17, 1994.

shown in Fig. 5. From these values we obtain tail radius of 12.5-10.6 $R_{E}$ for the average tail radius $\left(R_{0}=25 R_{E}\right)$ and external pressure $\left(P_{0}=0.032 \mathrm{nPa}\right.$ : corresponding to the average lobe magnetic field of $9.1 \mathrm{nT}$ ). Characteristics of magnetic field and plasma variations in the magnetotail are briefly described in the following:

Interval A: Magnitudes of magnetic field of larger than $40 \mathrm{nT}$ were observed associated with a relatively high density and tailward flowing plasma, although the magnetic field was relatively stable, indicating the lobe signature. This indicates that GEOTAIL observed cold dense ion flows or was in a magnetosheath-like boundary layer (Gosling et al., 1985; Siscoe et al., 1994; Frank and Paterson, 1994; Hirahara et al., 1995). The largest magnetic field in the magnetotail was registered at 0121:03.

Interval B: GEOTAIL first encountered the north lobe and then crossed the neutral sheet into the south lobe. The satellite again returned to the north lobe before the reentry into the magnetosheath. A bipolar signature was observed associated with the second crossing of the neutral sheet, indicating the passage of a plasmoid. Note that GEOTAIL was at a position far from the nominal equatorial plane, $Z^{\prime}=27 R_{E}$ on the duskside.

Interval C: In this interval magnetic field components showed the signature of the tail lobe, but density and bulk flow decreases were small. This indicates that GEOTAIL was in a high density boundary layer.

Interval E: A crossing from a north-to south lobe was observed. In this case the reentry into the magnetosheath was from the northern lobe as in case of interval B.

Interval F: This interval was not preceded by the southward sheath field. The transition from the sheath to the magnetotail is not well defined in the magnetic field as compared with the other cases. Sheath flows before this interval deviated duskward by approximately 15 degrees from the GSM axis. Thus, this excursion was mainly due to the orientation shift of the tail axis. A temperature increase after decreases of density around 0529 suggests that the spacecraft encountered a plasma sheet-like region just after the magnetopause crossing. Similar characteristics were also noted at the beginning of intervals $\mathrm{A}$ and $\mathrm{E}$. The density of the tail lobe was below $1 / \mathrm{cm}^{3}$ from 


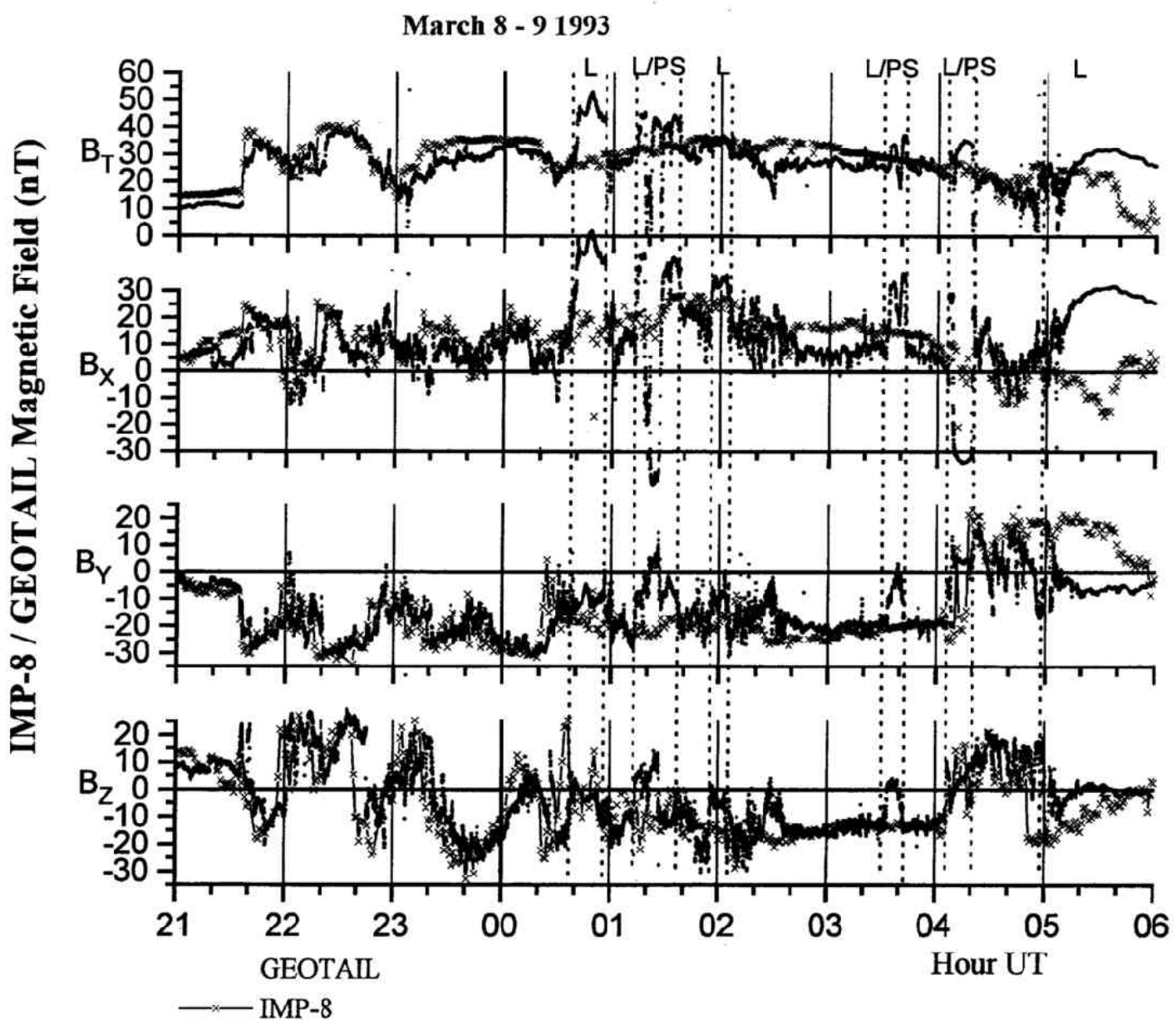

Fig. 4. Geotail and IMP-8 magnetic field data in GSM coordinates on March 8-9, 1993. Time for GEOTAIL data is shifted by 33 minutes taking into account an convection speed of IMF variations to the location of GEOTAIL. Dashed lines indicate magnetopause crossings of the GEOTAIL spacecraft. L and PS denote that GEOTAIL observed the lobe and the plasma sheet, respectively.

0550 to 0714 . The magnetic field intensity in the tail lobe dropped to $16 \mathrm{nT}$ at 0714 . The tail lobe was also observed after $08 \mathrm{~h}$ on March 9, and the magnitude of lobe field was less than 15 nT. These decreases in lobe field strength were associated with decreases in external pressure.

A common feature of flow direction in the magnetospheric intervals, except $\mathrm{C}$ and $\mathrm{F}$, is that plasma flows were slightly northward after the entry and were southward before the sheath entry. Also, flows were duskward during magnetospheric intervals. It is very likely that the tail boundary moved outward-then-inward during these intervals.

\subsection{April 17, 1994}

The largest magnetic storm during the deep tail epoch of GEOTAIL observations was observed on April 17, 1994. Since IMP 8 data are not available for this interval, we will refer to ground magnetic data as a measure of solar wind activity. The Dst index began to decrease around $01 \mathrm{~h}$ and reached the minimum, $\sim 280 \mathrm{nT}$, around 0740 (Iyemori et al., 1995). Figure 6 


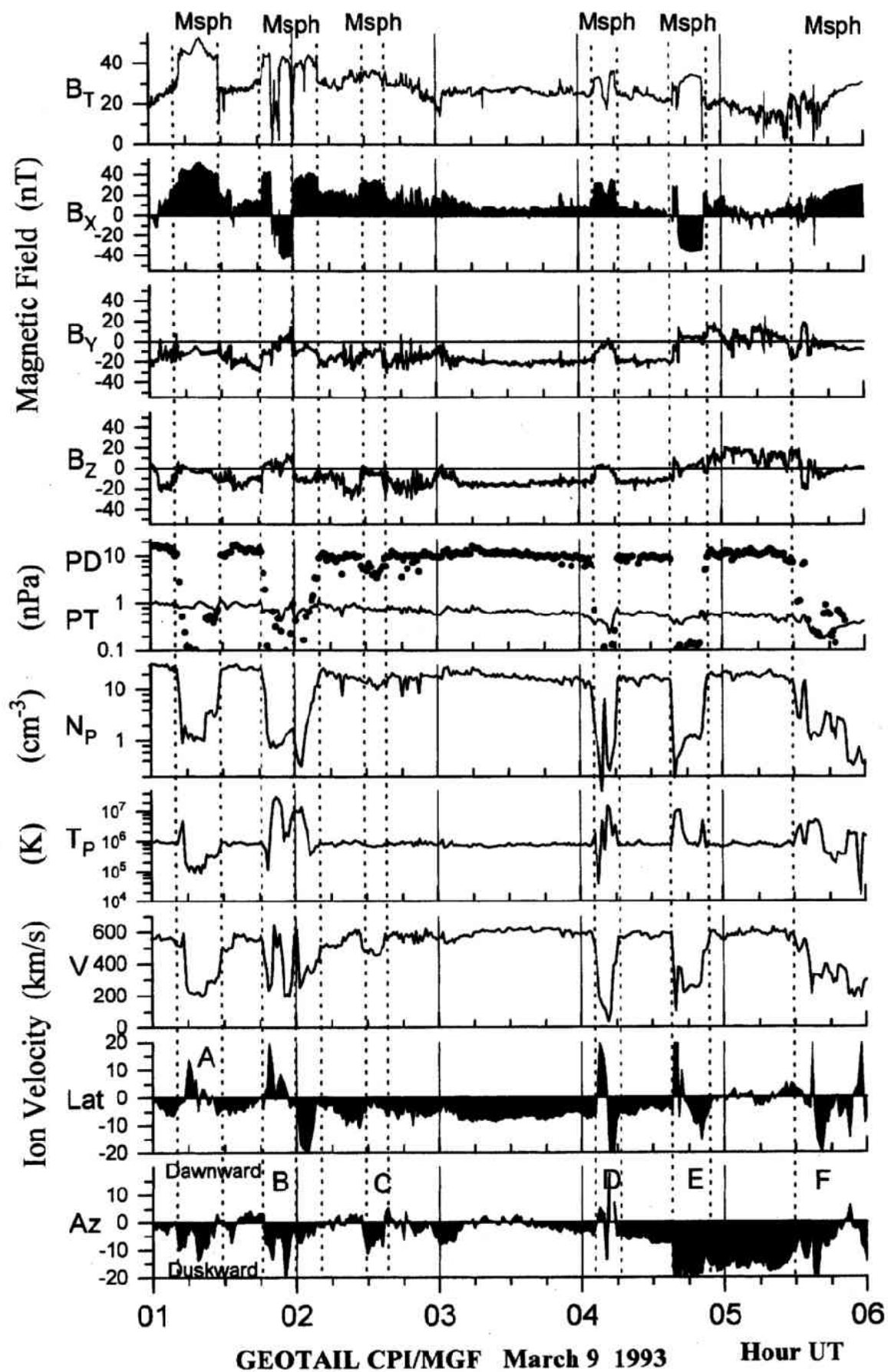

Fig. 5. Magnetic field data ( 3 sec.) are plotted along with plasma parameters, density $\left(N_{P}\right)$, temperature $\left(T_{P}\right)$, bulk speed $(V)$, latitude and azimuthal angles from $-X_{C S M}$ axis, from the CPI hot plasma detector. Dynamic pressure, $N_{P} m V^{2}(P D)$ and total pressure, $2 N_{p} k T_{p}+B_{T}^{2} / 2 \mu$ are also shown. 
April 171994
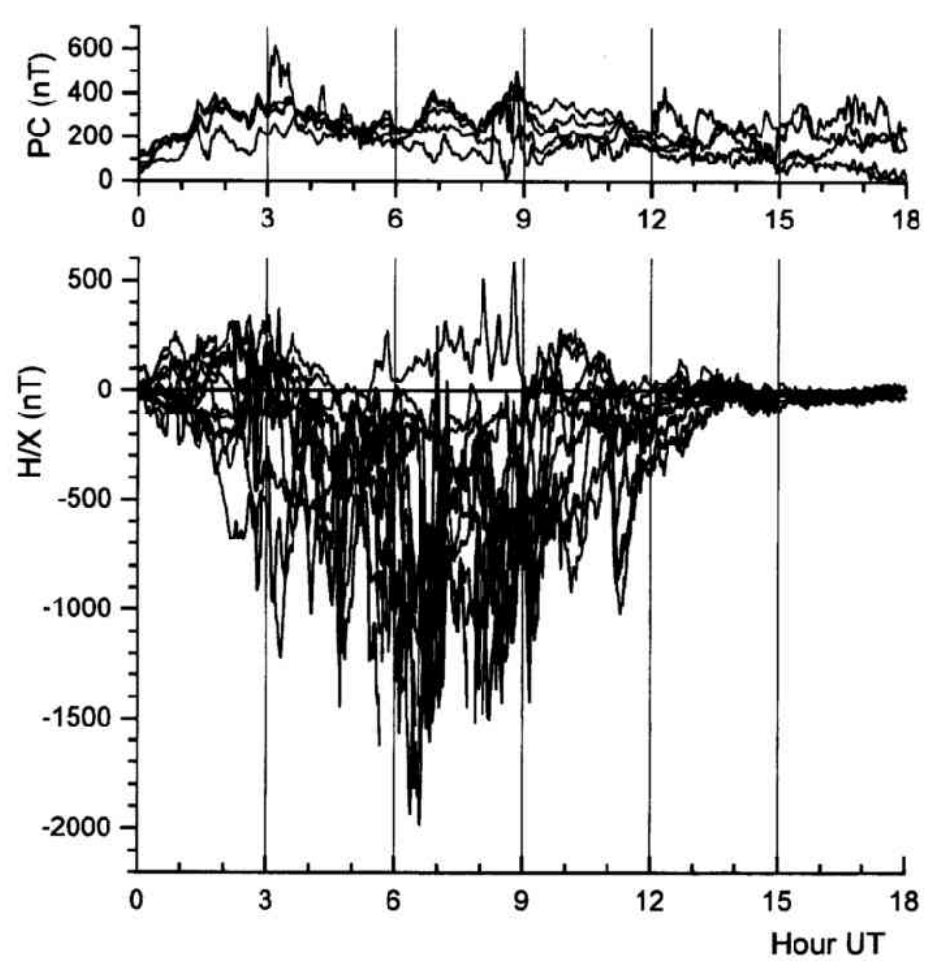

Fig. 6. Overlapping plots of magnetic field data from polar regions. $P C$ in the top panel indicates variations of $\left(d X m^{2}+d Y m^{2}\right)^{1 / 2}$, from Eureka $(88.9,318$ in geomagnetic coordinates), Resolute Bay $(84.5,316.0)$, Cambridge Bay $(77.6,306)$, Baker Lake $(74.5,325.5)$ and Ny lesund $(76.0,112.3)$, where d denotes deviation from the quiet day mean. In the bottom panel are plotted magnetic field data from 12 stations of the $210^{\circ}$ median and STEP polar networks.

shows ground magnetic activities in the polar region. In the bottom of the figure are overlapping plots of $X_{m}$ components from auroral and subauroral latitude stations. Magnitudes of horizontal disturbances in the polar cap, denoted by $P C$ in Fig. 6 , roughly represent the sunward current in the central polar cap. Large electrojet activities of more than $1000 \mathrm{nT}$ were observed at stations located around $60^{\circ}$ in geomagnetic latitude associated with the development of ring current activity. We also note significantly large activity of $200-400 \mathrm{nT}$ in the polar cap.

In Fig. 7 are plotted LEP/MGF data for a 15 hour interval during this magnetic storm sequence. Although GEOTAIL was near the central part of the nominal magnetotail, it was in the magnetosheath for more than half of this interval. Note that the $B_{z}$ component is dominant in the sheath and that the $B_{y}$ component is small in the first half of this interval. The magnetospheric signatures were observed in the following periods: 0010-0121, 0202-0212, 0225-0248, 0302-0307, 0314-0416, 0433-0439, 0612-0638, 1012-1240, and 1307-1322. After the last entry the spacecraft was in the sheath for about seven hours. The $B_{z}$ component in the sheath was strongly northward for this interval.

During magnetospheric intervals GEOTAIL observed the south lobe and/or the north lobe. An interesting feature to be noted here is a systematic tendency of plasma flow directions in the magnetotail. When the spacecraft was in the southern lobe, plasma flows had northward components. In the period of 0612-0638, GEOTAIL first entered the plasma sheet of high temperature 


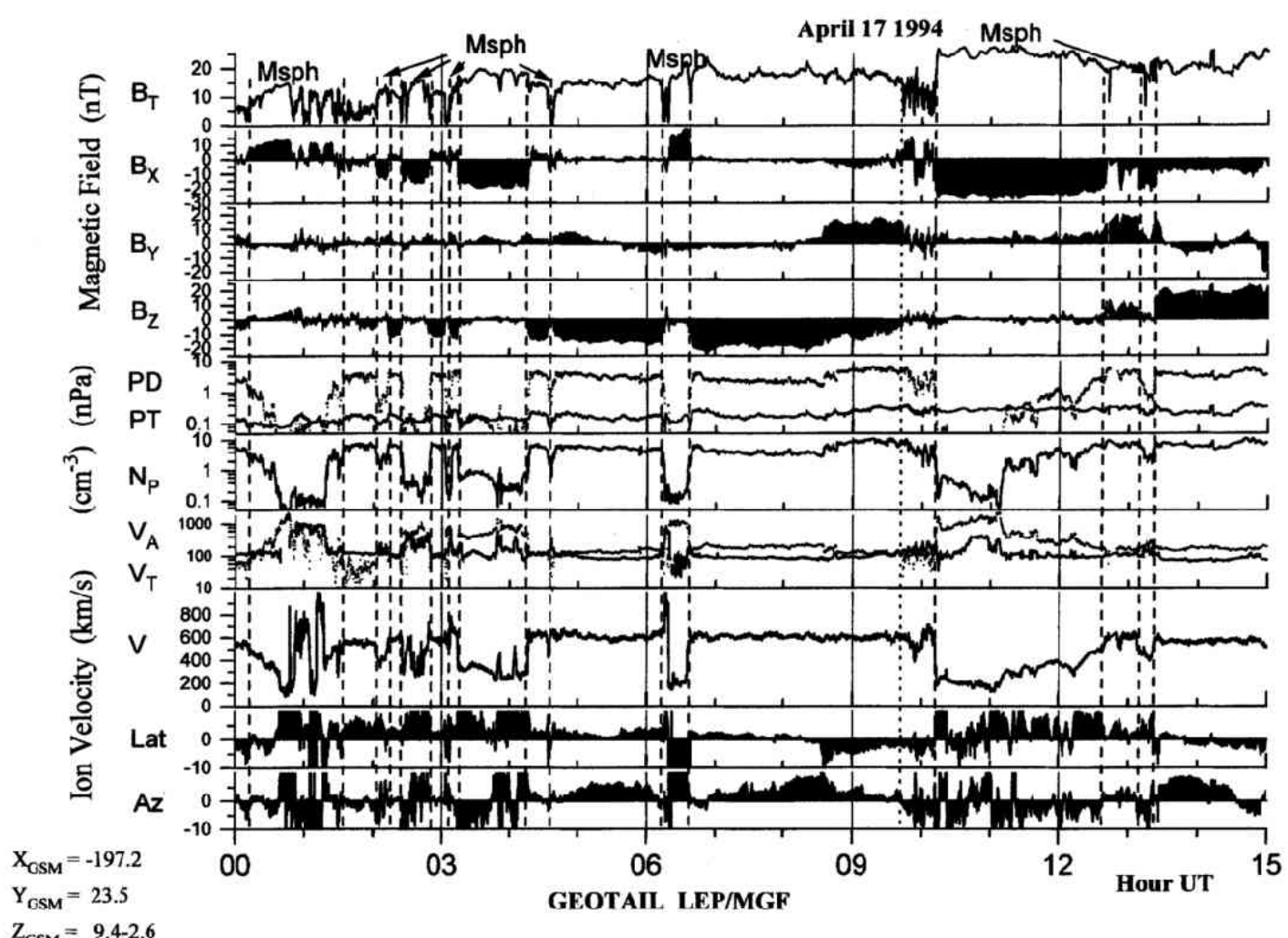

Fig. 7. Magnetic field and plasma parameters from the LEP experiment on April 17, 1994 are plotted for 15 hour interval in GSM coordinates, in a format similar to Fig. 5. The GEOTAIL spacecraft was at positions around $X_{G S M}=-197 R_{E}$. Time resolutions are $\sim 3$ seconds for magnetic field data and $\sim 12$ seconds (four spin periods) for plasma data, respectively. $V_{A}$ (dot) and $V_{T}$ (line) in the fourth panel from the bottom represent Alfven velocity and thermal velocity $(\mathrm{km} / \mathrm{s})$.

and tailward flows and then moved to the northern lobe. Southward and duskward components of plasma flows predominate in the northern lobe. Similar to the previous cases, magnetospheric periods were preceded by the southward sheath magnetic fields. It is seen that the magnitude of the $Z$-component of the sheath magnetic field gradually increased, corresponding to the development of Dst and substorms.

In order to examine how entries of the spacecraft relate to directional changes in sheath flow, we calculate distances from the expected center of the magnetotail, assuming the tail axis is parallel to the sheath flow measured with LEP. Figure 8 represents the radial distance of GEOTAIL from the estimated tail axis and the GEOTAIL location in $Y-Z$ plane based on sheath flows when the spacecraft was in the magnetosheath. In the bottom panel is also shown the radius of the tail calculated from the magnetosheath pressure, assuming the tail flux conservation.

The interesting feature to be noted is that radial distances show a change with a time scale of two hours or more, indicating the windsock effect (Shodhan et al., 1996). Spacecraft entries were observed when the radial distance is 20-30 $R_{E}$. Radial distances at times of magnetopause crossings are $5 R_{E}$ or more compared with tail radii estimated from the flux conservation. Other features to be noted are relation between the spacecraft location in the magnetotail and the $B_{y}$ component of magnetic field. GEOTAIL was mostly in the north lobe in period of $0612-0638$, 
April 171994

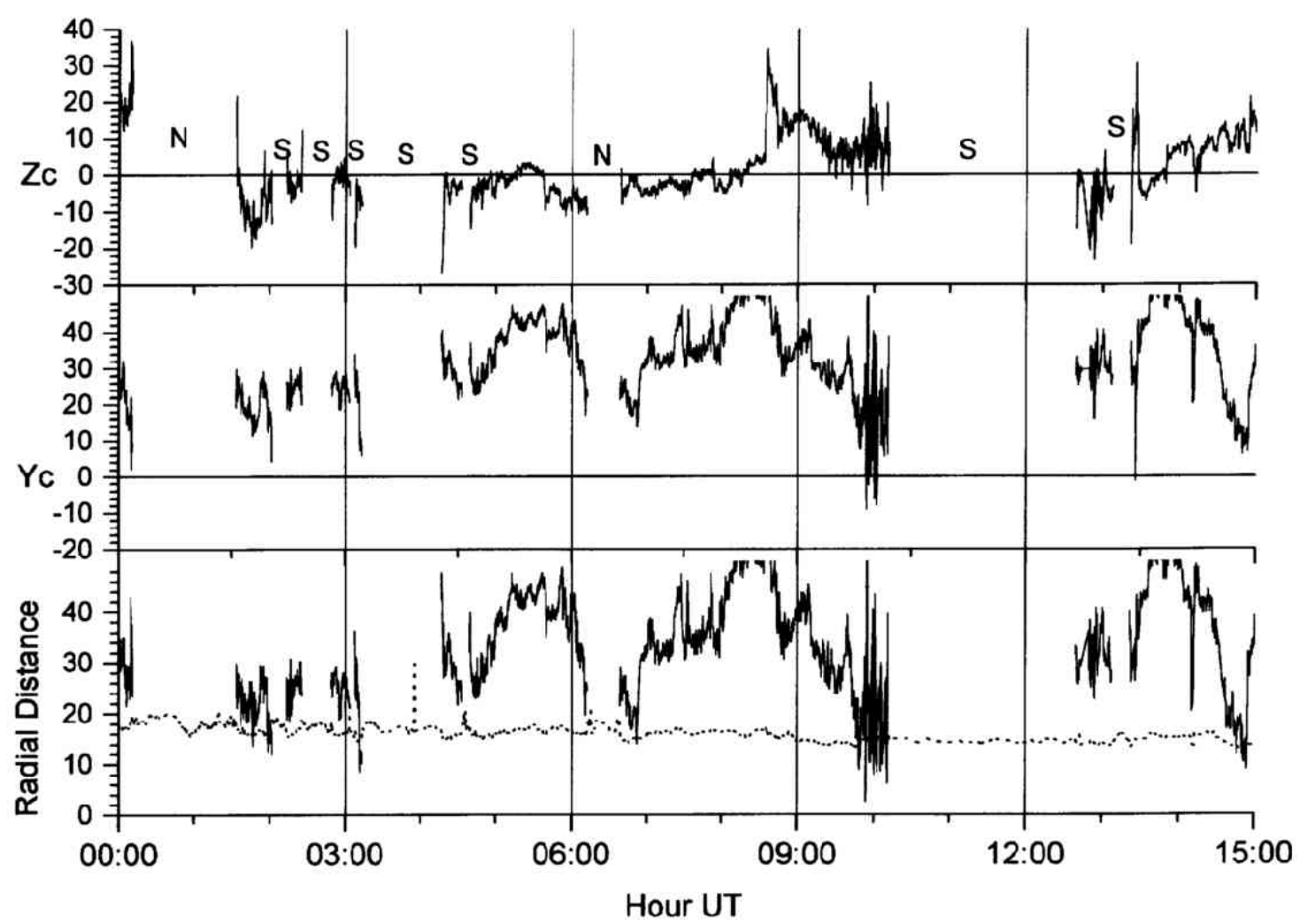

Fig. 8. Radial distances from the center of the magnetotail, and spacecraft locations in $Y-Z$ plane, assuming the tail axis is parallel to the sheath flow measured with LEP. In the bottom panel is shown the radius of the tail (dashed line) calculated from the flux conservation. $\mathrm{N}$ and $\mathrm{S}$ indicate spacecraft residences in the north and south lobes, respectively.

although the spacecraft was in the south of estimated ecliptic plane $\left(Z_{c}=-3 \sim-7 R_{E}\right)$. In periods before and after this interval $B_{y}$ components in the sheath were negative. The observed north lobe encounter is expected from the magnetotail twisting model. The model predicts that the sense of the torque from the negative $B_{y}$ is to raise the south lobe above the dawnside ecliptic plane and lower the north lobe below the duskside ecliptic plane (e.g. Sibeck et al., 1986). The south lobe encounter in the interval of 1012-1240 is also explained in terms of the $B_{y}$ effect. In this interval the spacecraft appears to be close to the ecliptic plane. $B_{y}$ components before and after this interval were strongly positive. Thus, the south lobe encounter is consistent with the twisting model.

In the period of $1012-1240$ we can see a good correlation between the density and flow speed, indication that the density gradually decreases with an increase in flow speed. Figure 9 illustrates relations between flow speed and density, thermal velocity and total magnetic field. A dotted line in this figure indicates an approximate boundary between the magnetotail and magnetosheath signatures. As is also evident from the plots in Fig. 7, the thermal velocity is almost constant, and the density increases with the increase in plasma flow for speeds of larger than $\sim 200 \mathrm{~km} / \mathrm{s}$. The field magnitude is in the range of 20-27 nT indicating the lobe signature. 


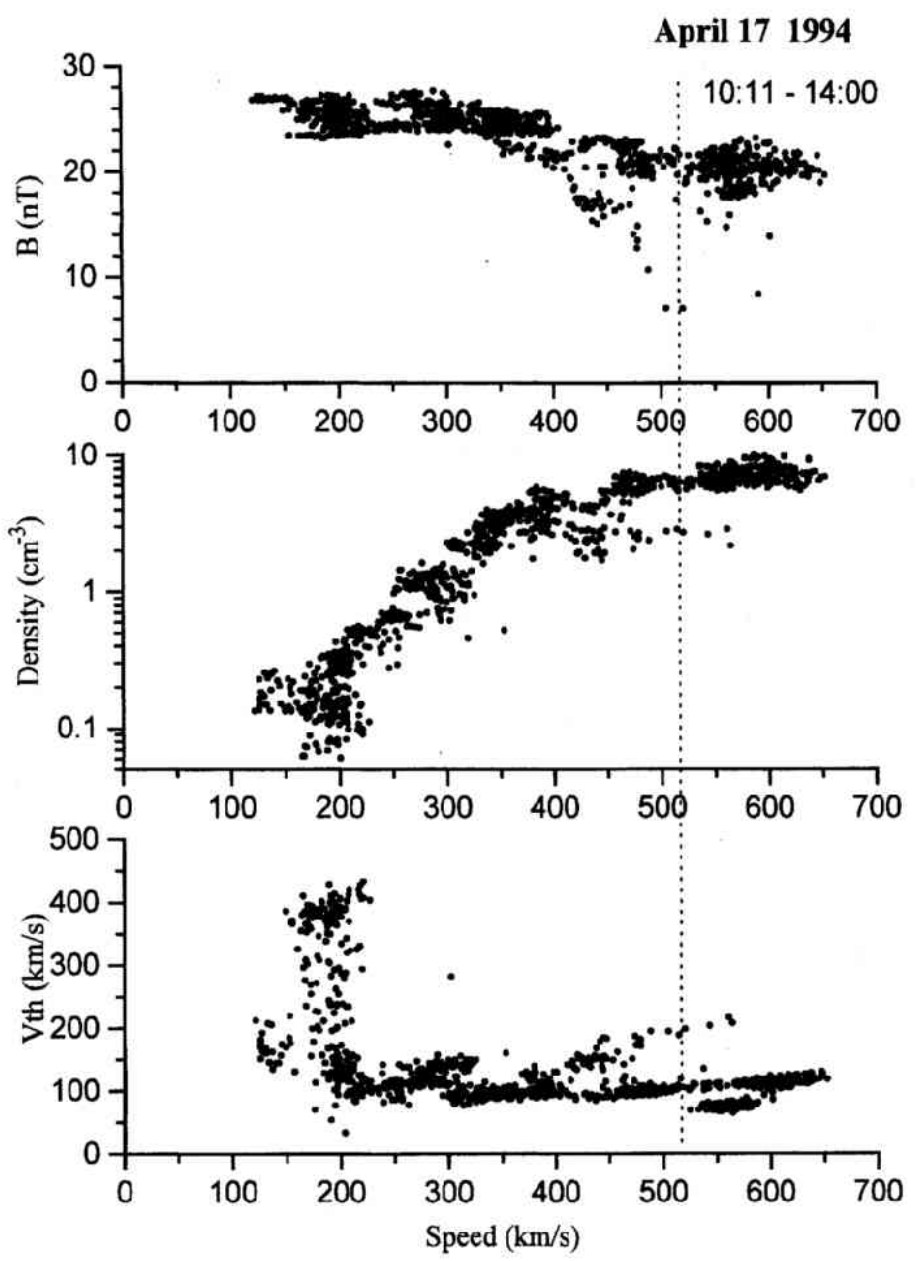

Fig. 9. Thermal velocity (Vth), density, and field magnitude variations versus plasma flow speed for the period of $1011-1400$.

These plasma parameters change smoothly at the magnetopause crossing around 1240 . This systematic relation appear to show spatial characteristics of the cold dense ion beam in the tail lobe (Mukai et al., 1994a; Hirahara et al., 1995; Siscoe et al., 1994). The transition from the tail lobe to the magnetosheath around 1240 was associated with a magnetic field rotation without a significant jump in magnitude, showing the characteristics of rotational discontinuity. This suggests the direct entry of sheath plasmas through the magnetopause (Mozer et al., 1994; Yamamoto et al., 1994b).

Since electrojet activity is too complicated to identify individual substorm onset, it is difficult to relate individual substorm onset to magnetospheric entries of GEOTAIL. The following correspondences between ground magnetic activities and excursions into the magnetotail are, however, noted here: several excursions from 0202 to 0433 would occur associated with the development of electrojets after about 0130 , since the tail orientation change is considered to be a slowly varying phenomenon with a time scale of more than two hours (Shodhan et al., 1996). The temporal entry after 0612 may correspond to maximum electrojet activity around $06 \mathrm{~h}$.

A sudden rotation of the sheath magnetic field occurred associated with an increase in ion 
density at 0834 . If this discontinuity propagates with a speed of approximately $600 \mathrm{~km} / \mathrm{s}$ as measured at GEOTAIL in the magnetosheath, it would pass by the earth around $08 \mathrm{~h}$. A sudden change is seen in the bottom panel of Fig. 6 in association with an increase in the polar cap activity. This suggests that the lobe entry from 1012 would relate to magnetic activity after $08 \mathrm{~h}$. Dst and substorm activities recovered rapidly associated with the northward excursion of $B_{z}$ component as observed by GEOTAIL after the last entry.

\section{Summary and Discussion}

In this paper we have examined intervals when the tail lobe field magnitude was larger than $20 \mathrm{nT}$, using magnetic field and plasma data obtained by the deep tail survey of the GEOTAIL spacecraft. These intervals were found to mostly correspond to the growing stage of ring current under enhanced solar wind conditions. We have shown two examples of large field events, one of which is the unusual case with the largest magnetic field of $53 \mathrm{nT}$ in the tail lobe. Although the analysis for other events is still under way, observations including other events are summarized as follows:

1) A series of temporal entries of the GEOTAIL spacecraft into the magnetotail at positions of $100 R_{E}$ downstream from the earth are observed during the growing stage of ring current. The time scale of these intervals is typically $\sim 20$ minutes.

2) The magnitude of the lobe magnetic field during these magnetospheric residences is usually two or more times the average lobe magnetic field of $\sim 9 \mathrm{nT}$. The largest magnitude observed by GEOTAIL was $53 \mathrm{nT}$.

3) Spacecraft entries into the magnetotail are often preceded by the negative sheath magnetic field, as expected from the fact that magnetic storms occur in association with sustained intervals of the IMF negative $B_{z}$ component.

4) It is confirmed that the pressure balance is approximately holds at times of magnetopause crossings during enhanced sheath conditions. This indicates that the strong lobe fields are due to the compression associated with the enhanced solar wind parameters.

5) Cold ion flows of $200-300 \mathrm{~km} / \mathrm{s}$ with densities of $0.5-1$ particles $/ \mathrm{cm}^{3}$ are observed in the lobe region.

The solar wind flow direction should be a significant factor in whether or not the spacecraft observes the tail lobe at a distance of larger than the average cross-sectional radius of the tail (Fairfield, 1993). The size of the tail radius is another parameter that will determine whether or not a spacecraft observes the tail. Nakamura et al. (1996) have discussed the probability of encountering magnetopause signatures near the nominal center of the magnetotail, based on GEOTAIl data obtained during a weak storm on January 25, 1993. They have concluded that solar wind variations, which cause magnetic storm activity, significantly change the orientation as well as the size of the distant tail so that the magnetosheath can be observed near the average center of the magnetotail. We also examined the April 17 event, assuming the tail axis is parallel to the magnetosheath flow as was done by Nakamura et al. (1996). Inferred tail radii at times of magnetopause crossings are larger than those estimated from the tail flux conservation. In cases of March 9, tail radii at times of magnetopause crossings estimated from the same procedure range from 14 to $42 R_{E}$ for GEOTAIL plasma data and from 10 to $23 R_{E}$ for IMP 8 plasma data. These values are considerably larger than those from the flux conservation as mentioned before. However, tail radii estimated from GEOTAIL and IMP 8 data are not necessarily consistent with each other. Since simulations of Frank et al. (1995) suggest that the $Y$ and $Z$ flow velocities near the boundary depend on position, the sheath flow may not a good substitute for the solar wind when the $B_{y}$ component is large as in case of March 9 event. We will further examine the unusual case on March 9, 1993 to clarify the discrepancy between IMP 8 and GEOTAIL. We have also calculated tail radii at times of magnetopause crossings for other events and obtained 
a tendency that estimated values are larger than those inferred from the flux conservation. It is suggested that the dayside merging of southward IMF contributes an increase of magnetic flux in the distant tail down to about $-200 R_{E}$ during disturbed periods. This inference is supported by the well-known fact that the polar cap becomes larger associated with the development of ring current.

In recent years a number of global magnetohydrodynamic(MHD) simulation models have been developed for the interaction of solar wind with the magnetosphere (Usadi et al., 1993; Walker et al., 1993; Ogino et al., 1994; Frank et al., 1995). Among them, Frank et al. (1995) have reported the first direct comparison of in situ observations of plasma and magnetic fields in the distant magnetotail with a time-dependent MHD simulation. In this study simultaneous observations of the solar wind ions and the IMF with the IMP 8 spacecraft upstream from the earth during a moderately active period, when IMF $B_{y}$ and $B_{z}$ components were fluctuating with a time scale of 30 minutes, were used as the driving input for a global MHD model. These observations were obtained with GEOTAIL at a downstream distance of $81 R_{E}$ near the dawnside magnetopause. The magnetopause positions encountered at this time was examined by using energetic particle data (Williams et al., 1994). Williams et al. found that the unusual magnetopause positions could be explained in part by solar wind aberration effects and suggested that additional processes, such as magnetospheric breathing modes, are required to fully explain these observations. Our observations also suggest that internal processes related to substorms are needed to account for intermittent and temporal entries of the spacecraft into the magnetotail of which time scale is typically 20 minutes.

In the model simulation by Frank et al. (1995), the flow velocity of the solar wind is fixed along the sun-earth line. The results indicate that the agreement between the computed and observed magnetic fields is sufficiently good. This means that magnetotail encounters observed by a single point measurement results from twisting and associated deformation of the tail cross section due to the torque exerted by the $B_{y}$ component. Frank et al. (1995) have concluded that the overall agreement of the modeled results and the observations for a single position in the vicinity of the distant magnetopause provides ample motivation to improve and further verify the robustness of the model. In order to understand the large-scale structure and complicated dynamics of the magnetotail based on a single point measurement during disturbed conditions, comparison with global MHD models should be further proceeded.

The work at Nagoya University was supported by a grand-in-aid for scientific research project 05452082, Ministry of Education Science, Sports and Culture, Japan. The research at The University of Iowa was supported in part by NASA grant NAG5-2371. The IMP 8 data were made available from the LEP NASA/GSFC data processing team and the MIT Space Plasma Physics Group. We would like to thank one of referees for critical and helpful comments on the paper.

\section{REFERENCES}

Baker, D. N., R. C. Anderson, R. D. Zwickle, and J. A. Slavin, Average plasma and magnetic filed variations in the distant magnetotail associated with near-Earth substorm effects, J. Geophys. Res., 92, 71-81, 1987.

Cowley, S. W. H., Magnetospheric asymmetries associated with the $Y$-component of the IMF, Planet. Space Sci., 29, 79-96, 1981.

Fairfield, D. H., Solar wind control of the distant magnetotail: ISEE 3, J. Geophys. Res., 98, 21,265-21,276, 1993.

Fairfield, D. H., R. P. Lepping, L. A. Frank, K. L. Ackerson, W. R. Paterson, S. Kokubun, T. Yamamoto, K. Tsuruda, and M. Nakamura, Geotail observations of an unusual magnetotail under very northward IMF conditions, J. Geomag. Geoelectr., 48, this issue, 1996.

Frank, L. A., K. L. Ackerson, W. R. Paterson, J. A. Lee, M. R. English, and G. L. Picket, The comprehensive plasma instrumentation (CPI) for the GEOTAIL spacecraft, J. Geomag. Geoelectr., 46, 23-37, 1994.

Frank, L. A. and W. R. Paterson, Survey of electron and ion bulk flows in the distant magnetotail with the Geotail spacecraft, Geophys. Res. Lett., 21, 2963-2966, 1994. 
Frank, L. A., M. Ashour-Abdalla, J. Berchem, J. Raeder, W. R. Paterson, S. Kokubun, T. Yamamoto, R. P. Lepping, F. V. Coronitti, D. H. Fairfield, and K. L Ackerson, Observations of plasma and magnetic fields in Earth's distant magnetotail: Comparison with a global MHD model, J. Geophys. Res., 100, 19,177-19,190, 1995.

Gosling, J. T., D. N. Baker, S. J. Bame, W. C. Feldman, R. D. Zwickle, and E. J. Smith, North-south and dawn-dusk plasma a symmetries in the distant tail lobes: ISEE-3, J. Geophys. Res., 90, 6354-6360, 1985.

Hirahara, M., T. Mukai, S. Machida, Y. Saito, T. Terasawa, T. Yamamoto, and S. Kokubun, Cold dense ion flows with multiple components observed in the distant tail lobe by GEOTAIL, J. Geophys. Res., 1995 (in press).

Iyemori, T., T. Araki, T. Kamei, and M. Takeda, Mid-latitude geomagnetic indices ASY and SYM, No. 5, Kyoto University, April 1995.

Kokubun, S., T. Yamamoto, M. Acu a, K. Hayashi, K. Shiokawa, and H. Kawano, The GEOTAIL magnetic field experiment, J. Geomag. Geoelectr., 46, 7-21, 1994a.

Kokubun, S., Y. Kamide, R. Nakamura, T. Yamamoto, K. Tsuruda, T. Mukai, A. Nishida, L. A. Frank, and W. R. Paterson, Structure of the distant magnetotail during the main phase of magnetic storm, EOS, 75, 538, $1994 \mathrm{~b}$.

Mozer, F. S., H. Hayakawa, S, Kokubun, M. Nakamura, T. Okada, T. Yamamoto, and K. Tsuruda, Geophys. Res. Lett., 21, 2959-2912, 1994.

Mukai, T., M. Hirahara, S. Machida, Y. Saito, T. Terasawa, and A. Nishida, Geotail observation of cold ion streams in the medium distant magnetotail lobe in a course of a substorm, Geophys. Res. Lett., 21, 1023-1026, 1994a.

Mukai, T., S. Machida, Y. Saito, M. Hirahara, T. Terasawa, N. Kaya, T, Obara, M. Ejiri, and A. Nishida, The low energy particle (LEP) experiment onboard the GEOTAIL Satellite, J. Geomag. Geoelectr., 46, 669-692, 1994b.

Nakamura, R., S. Kokubun, Y. Kamide, T. Yamamoto, L. A. Frank, and W. R. Paterson, Observations of magnetosheath near the center of the magnetotail during a weak geomagnetic storm on January 25, 1993, J. Geomag. Geoelectr., 48, this issue, 1996.

Ogino, T., R. J. Walker, and M. Ashour-Abdalla, A global magnetohydrodynamic simulation of the response of the magnetosphere to a northward turning of the interplanetary magnetic filed, J. Geophys. Res., 99, 11,027$11,042,1994$.

Owen, C. J., J. A. Slavin, I. G. Richardson, N. Murphy, and R. J. Hunds, Average motion, structure and orientation of the distant magnetotail determined from remote sensing of the edge of the plasma sheet boundary layer with $E>35 \mathrm{keV}$ ions, J. Geophys. Res., 100, 185-204, 1995.

Shodhan, S., G. L. Siscoe, L. A. Frank, K. L. Ackerson, and W. R. Paterson, Boundary oscillation at Geotail: Windsock, breathing, and wrenching, J. Geophys. Res., 101, 2577-2586, 1996.

Sibeck, D. G., G. L. Siscoe, J. A. Slavin, E. J. Smith, B. T. Tsurutani, and R. P. Lepping, The distant magnetotail's response to a strong interplanetary magnetic field $B_{y}$ : Twisting, flattening, and filed line bending, J. Geophys. Res., 90, 4011-4019, 1985a.

Sibeck, D. G., G. L. Siscoe, J. A. Slavin, and R. P. Lepping, Major flattening of the distant geomagnetic tail, J. Geophys. Res., 90, 4223-4237, 1985 b.

Sibeck, D. G., J. A. Slavin, E. J. Smith, and B. T. Tsurutani, Twisting of the geomagnetic tail, in Solar WindMagnetosphere Coupling, edited by Y. Kamide and J. A. Slavin, pp. 731-738, Terra Scientific Pub., Tokyo, 1986.

Siscoe, G. L., L. A. Frank, K. L. Ackerson, and W. R. Paterson, Properties of the mantle-like boundary layer: Geotail data compared with a mantle model, Geophys. Res. Lett., 21, 2975-2978, 1994.

Slavin, J. A., E. J. Smith, D. G. Sibeck, D. N. Baker, R. D. Zwickle, and S.-I. Akasofu, An ISEE 3 study of average and substorm conditions in the distant magnetotail, J. Geophys. Res., 90, 10,875-10,895, 1985.

Usadi, A., A. Kageyama, K. Watanabe, and T. Sato, A global simulation of the magnetosphere with a long tail: Southward and northward interplanetary magnetic field, J. Geophys. Res., 98, 7503-7517, 1993.

Walker, R. J., T. Ogino, J. Raeder, and M. Ashour-Abdalla, A global magnetohydrodynamic simulation of the magnetosphere when the interplanetary magnetic field is southward: The onset of magnetotail reconnection, J. Geophys. Res., 98,17,235-17,249, 1993.

Williams, D. J., A. T. Y. Lui, R. W. McEntire, V. Angelopoulos, C. Jacquey, S. P. Christon, L. A. Frank, K. L. Ackerson, W. R. PATERSON, S. Kokubun, T. Yamamoto, and D. H. Fairfield, Magnetopause encounters in the magnetotail at distances of $\sim 80 R_{e}$, Geophys. Res. Lett., 21, 3007-3010, 1994.

Yamamoto, T., K. Shiokawa, and S. Kokubun, Magnetic field structures of the magnetotail as observed by Geotail, Geophys. Res. Lett., 21, 2875-2878, 1994a.

Yamamoto, T., A. Matsuoka, K. Tsuruda, H. Hayakawa, A. Nishida, M. Nakamura, and S. Kokubun, Dense plasmas in the distant magnetotail as observed by Geotail, Geophys. Res. Lett., 21, 2879-2882, 1994b.

Yumoto, K., Y. Tanaka, T. Oguti, K. Shiokawa, Y. Yoshimura, A. Isono, B. J. Fraser, F. W. Menk, J. W. Lynn, M. Seto, and $210^{\circ} \mathrm{MM}$ Magnetic Observation Group, Globally coordinated magnetic observations along $210^{\circ}$ magnetic meridian during STEP Period: 1. Preliminary results of low-latitude Pc 3's, J. Geomag. Geoelectr., 44, 261-276, 1992. 